\title{
ASCI 2010 standardized practice protocol for cardiac magnetic resonance imaging: a report of the Asian society of cardiovascular imaging cardiac computed tomography and cardiac magnetic resonance imaging guideline working group
}

\author{
ASCI CCT and CMR Guideline Working Group • Carmen W. S. Chan • \\ Byoung Wook Choi · Masahiro Jinzaki $\cdot$ Kakuya Kitagawa $\cdot$ I-Chen Tsai • \\ Hwan Seok Yong • Wei Yu
}

Received: 29 August 2010/Accepted: 15 September 2010/Published online: 6 October 2010

(C) The Author(s) 2010. This article is published with open access at Springerlink.com

\begin{abstract}
These practice guidelines are recommended by the Asian Society of Cardiovascular Imaging (ASCI), the sole society in Asia designated for cardiovascular imaging, to provide a framework to healthcare providers for suggested essential elements in cardiac magnetic resonance (CMR) examinations of different disease spectra. The guideline is composed of recommendations on the general technique, acquisition of some basic modules, and protocols on stress tests. The protocols for specific diseases are provided in a table format for quick reference to be easily utilized for everyday clinical CMR.
\end{abstract}

\section{W. S. Chan $(\bowtie)$}

Division of Cardiology, Department of Medicine, Queen Mary Hospital, 102 Pok Fu Lam Road, Hong

Kong, China

e-mail: carmen.ws@gmail.com

B. W. Choi

Department of Radiology, Research Institute of

Radiological Science, Severance Hospital, Yonsei

University Health System, Seoul, Korea

\section{Jinzaki}

Department of Diagnostic Radiology,

Keio University School of Medicine,

Tokyo, Japan
Keywords Cardiac magnetic resonance imaging . Practice guideline $\cdot$ Stress protocol $\cdot$ ASCI $\cdot$ Asia
Abbreviations
ACLS Advanced cardiac life support
ASCI Asian society of cardiovascular imaging
CMR Cardiac magnetic resonance
ECG Electrocardiogram
ETL Echo train length
FSL Fast spin echo
GRE Gradient echo
GRE-EPI Gradient echo-echo planar imaging
HOCM Hypertrophic obstructive cardiomyopathy

\author{
K. Kitagawa \\ Department of Diagnostic Radiology, Mie University \\ School of Medicine, Tsu, Japan \\ I.-C. Tsai \\ Department of Radiology, Taichung Veterans General \\ Hospital, Taichung, Taiwan \\ H. S. Yong \\ Department of Radiology, Korea University Guro \\ Hospital, Seoul, Korea \\ W. Yu \\ Department of Radiology, Beijing Anzhen Hospital, \\ Capital Medical University, Beijing, China
}




$\begin{array}{ll}\text { IR } & \text { Inversion recovery } \\ \text { LGE } & \text { Late gadolinium enhancement } \\ \text { LPA } & \text { Left pulmonary artery } \\ \text { LV } & \text { Left ventricle } \\ \text { LVOT } & \text { Left ventricular outflow tract } \\ \text { MPA } & \text { Main pulmonary artery } \\ \text { MR } & \text { Magnetic resonance } \\ \text { PA } & \text { Pulmonary artery } \\ \text { PC } & \text { Phase contrast } \\ \text { RPA } & \text { Right pulmonary artery } \\ \text { RF } & \text { Radio frequency } \\ \text { RR } & \text { Relative risk } \\ \text { RV } & \text { Right ventricle } \\ \text { RVOT } & \text { Right ventricle outflow tract } \\ \text { SSFP } & \text { Steady state free precession } \\ \text { TE } & \text { Echo time } \\ \text { TR } & \text { Repetition time } \\ \text { TI } & \text { Inversion time }\end{array}$

\section{Introduction}

As the healthcare system, resource allocation, and pattern of diseases $[1,2]$ in Asia are different from those in Western Society, this practice protocol is recommended by the Asian Society of Cardiovascular Imaging (ASCI), the sole society dedicated to cardiovascular imaging in Asia, to provide a frame work for the suggested essential elements in cardiac magnetic resonance (CMR) examinations.

It must be emphasized that this guideline has been built on previously published guidelines from various professional societies [3-6], but is customized for CMR practice in Asia with its unique characteristics and where thalassemia [7-9] and valvular heart disease are common. The ultimate decision regarding the propriety of any specific procedure must be made after mutual communication with the referring physicians; the understanding of individual patient's condition; and the availability of resources, knowledge and technology provided by their respective centers by the responsible individuals participating and performing the CMR procedures.

The practice protocol starts with recommendations on the general technique and is followed by techniques on the acquisition of some basic modules. Recommended practice protocols on stress tests with separate checklists and monitoring sheets are provided. Protocols for specific diseases are summarized in a table format for quick reference.

\section{Guidelines}

Recommended practice protocol for CMR examination

\section{Patient preparation}

1. Check for any contraindication for MR examination, stress study, and contrast administration, if needed.

2. Obtain informed consent for the MR examination.

3. Advise patient on fasting before examination. This is not mandatory, but is advised.

4. Have patient stop intake of caffeine-containing food and beverages, theophylline or dipyridamole at least $24 \mathrm{~h}$ before adenosine study; avoid beta-blocker and nitrates for dobutamine study.

5. Educate and provide practice to patients on the breathing instruction.

6. Provide the patient with ear plugs.

7. Prepare the skin of the chest and/or abdomen for optimal attachment of electrodes for cardiac and respiratory gating.

8. Position the patient in the best comfortable supine position. Have the patient place the arms above the head to decrease wrap artifact. It is acceptable to put the arms along the side or crossed over the chest if the patient cannot tolerate arms overhead for extended periods of time.

\section{Equipment and safety}

1. Monitoring equipment (blood pressure, electrocardiogram, intercom communication with patients)

2. Resuscitation facilities :

i. emergency resuscitation policy in place

ii. availability of resuscitation team with medical/nursing staff having ACLS accreditation

iii. logistics for transferring patient to designated resuscitation area outside scanner

iv. resuscitation trolley with full set of emergency medications and equipment 
v. external pacer and defibrillator

vi. oxygen supply

vii. beta-blockers, nitrates, and aminophylline at hand

3. Stress MRI testing.

Detail on safety and equipment needed is listed in the performance protocol for the adenosine and dobutamine studies

Recommended practice protocol on basic modules

See Fig. 1 for a quick reference on the acquisition of major imaging planes described below.

\section{Left ventricular $(L V)$ structure and function module}

All scans are recommended to be performed as breathhold, multiphase steady state free precession
(SSFP) imaging at the end of the expiratory phase using all elements on the cardiac coil. All 17 segments of the heart can be covered by a combination of three short axis and two long axis views (four chambers and two chambers). Scout imaging-multistack three plane localizers: transtransaxial, coronal, sagittal

1. Calibrate for parallel imaging technique, if it is to be used.

2. Shimming

3. Transaxial $(8-10 \mathrm{~mm})$ set of SSFP or fast spin echo (FSE) images through the chest.

4. Vertical long axis scout cine images

i. The vertical long axis scout cine plane is prescribed from the axial localizer at the level of mitral and tricuspid valves.

ii. The slice through the LV apex and centre of the mitral valve is prescribed.

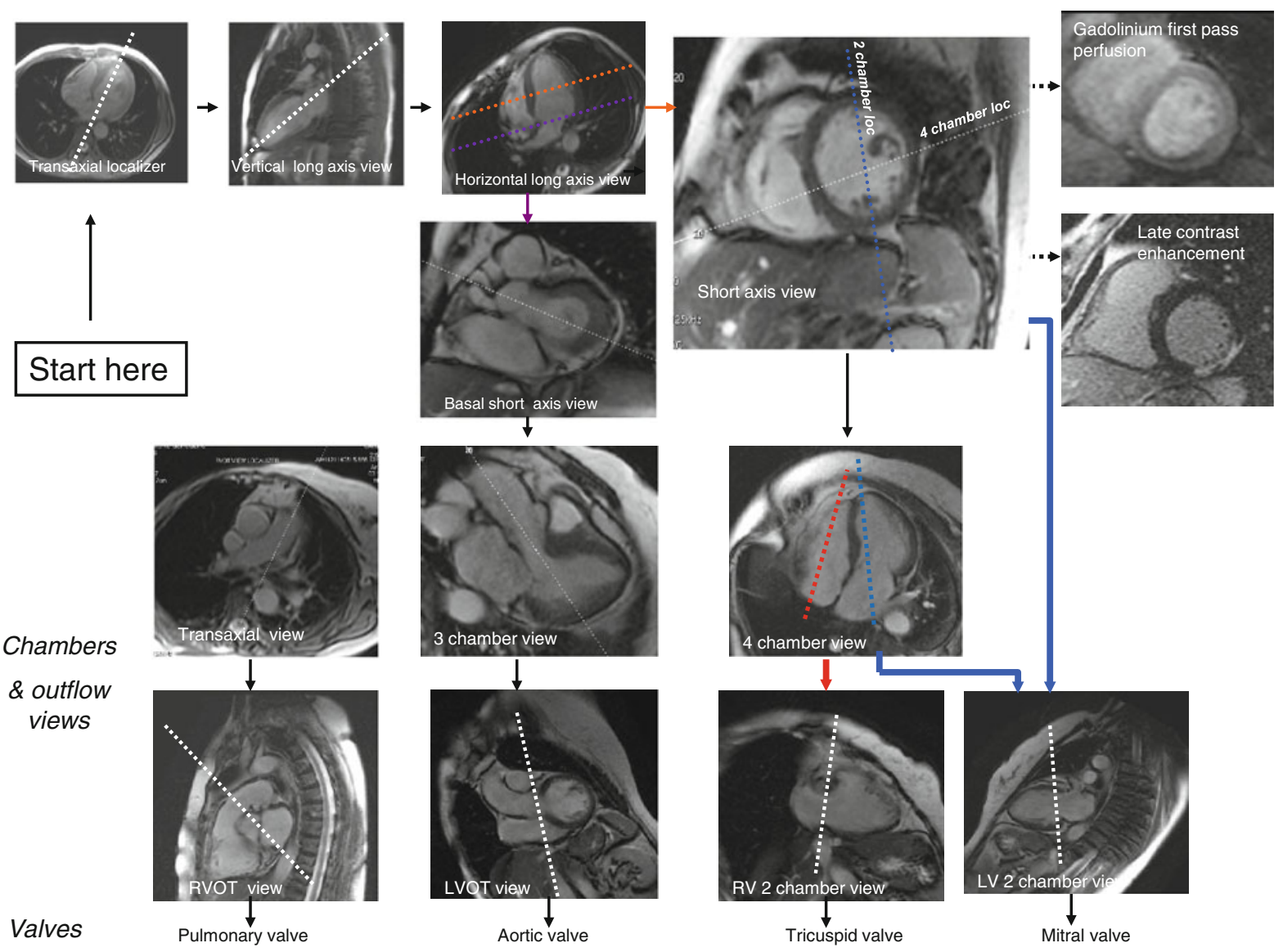

Fig. 1 A road map for major imaging planes of the cardiac magnetic resonance study 
5. Horizontal long axis scout cine images.

The horizontal long axis scout cine plane is prescribed from the end systolic phase of the vertical long axis scout cine image.

i. Position the slice at the center of the mitral valve and angulate through the LV apex.

ii. Scroll through the diastolic images to ensure the left atrium is included and properly imaged.

6. Short axis cine images.

The short axis cine planes are prescribed from previously acquired long axis scout cines at diastole, starting from the mitral valve plane through the LV apex, covering the whole ventricle perpendicular to the interventricular septum on horizontal long axis scout cine images.

i. Slice thickness 6-8 $\mathrm{mm}$; no gap

ii. Temporal resolution $\leq 45 \mathrm{~ms}$ between phases

iii. Parallel imaging to shorten scanning time

7. Other long axis cine images

i. The four chamber cine plane is prescribed from the vertical long axis scout cine and short axis cine images. The cut plane should pass through the LV apex and centre of mitral valve on the vertical long axis scout cine image. On the short axis cine image, angulate the cut plane so that it passes through the center of the LV chamber and lower corner of the RV border sure to avoid bisecting the LV outflow tract in the basal short axis view.

ii. The two chamber cine image is prescribed by a cut plane that bisects the LV apex and centre of the mitral valve in the four chamber view, as well as passing through the mid LV chamber in the short axis view. The cut plane needs to be in parallel to the ventricular septum in both views.

iii. The three chamber cine image is prescribed by a cut plane parallel to the long axis with the bisecting mitral valve and apex as well as the bisecting LV outflow tract in basal short axis view.

iv. The LV outflow tract (LVOT) long axis is obtained in the oblique coronal plane from the true axial scout images by positioning the slice through the aortic root and directing it toward the LV apex.

8. Additional views of cine images.

A contiguous stack of transaxial steady state free precession cine images from the top of the aortic arch to the inferior wall of the LV is recommended for congenital heart disease.

Right ventricular $(R V)$ structure and function module

$\mathrm{RV}$ short axis views are acquired in a similar fashion to the LV structure and function module. Use a transaxial stack of cines covering the RV for best identification of the tricuspid valve plane.

1. Right ventricular two chamber long axis view

i. Obtain the right ventricular two chamber long axis images from four chamber images

ii. Position the centre of the slice in the middle of the tricuspid valve and angulate through the RV apex. Scroll through phases to ensure the slice passes through the end-systolic and end-diastolic RV apex of the four chamber images.

2. Right ventricular outflow view (RVOT).

Prescribe by placing the slice through the centre of main pulmonary artery and the RV apex through a set of axial images.

3. Right ventricular inflow and outflow view.

An inflow/outflow plane of the RV can be acquired using a three-point plane.

i. Place the first point on the tricuspid valve of the four chamber view.

ii. Place the second point on the RV apex of the two chamber RV view.

iii. Place the third point on the pulmonary valve of the RVOT view.

\section{Gadolinium first pass perfusion module}

1. Use scout imaging as per the LV structure and function module.

2. Use saturation-recovery imaging with gradient echo-echo planar imaging (GRE-EPI) hybrid, GRE, or SSFP readout. 
3. Use at least three short axis slices per each heart beat (basal, mid, and apical level of LV). Add one slice in four chamber (for sepal/lateral segment ischemia) or two chamber view (for anterior/inferior segment) if the length of the RR interval permits.

4. Acquire the apical short axis slice first and the most basal short axis last during the cardiac cycle to minimize cardiac motion artifacts.

5. Use a slice thickness of $8-10 \mathrm{~mm}$, in-plane resolution, $\sim<3 \mathrm{~mm}$; twofold acceleration and temporal resolution $<100 \mathrm{~ms}$ or shorter.

6. Do a dry run at the end expiration for 5-10 phases to check and correct any position and phase wrap artifacts.

7. Use the following parameters:

i. Give contrast $(0.05-0.1 \mathrm{mmol} / \mathrm{kg}, 3-7 \mathrm{ml} / \mathrm{s})$ followed by at least $30 \mathrm{ml}$ saline flush $(5-7 \mathrm{ml} / \mathrm{s})$

ii. Start breathhold during early phases of contrast infusion before contrast reaches the LV cavity.

iii. Readout for 50-60 heart beats to capture the contrast wash-in and wash-out through LV myocardium.

\section{Late gadolinium enhancement ( $L G E$ ) module}

1. Wait for $10 \mathrm{~min}$ after administration of cumulative dose of $0.1-0.2 \mathrm{mmol} / \mathrm{kg}$ gadolinium. A shorter delay time may be considered if lower doses of contrast are used.

2. Use $2 \mathrm{D}$ segmented inversion recovery GRE imaging during the end of the diastolic phase.

3. Prescribe the slices at the identical location, views, and thicknesses as for cine imaging (short- and long-axis views).

4. In-plane resolution, $\sim 1.4-1.8 \mathrm{~mm}$; adjust acquisition duration to a shorter RR interval in tachycardia.

5. Set inversion time to null normal myocardium.

6. Adjust the read-out according to heart rate. Every other heart beat in normal setting of heart rate; every heart beat in the setting of bradycardia, and every third heart beat in the setting of tachycardia or arrhythmia.

\section{T2-weighted imaging}

1. Consider using either body coil or functional surface coil intensity correction algorithms.

2. Use breathhold, pre-contrast segmented FSE imaging (double inversion recovery).

3. Select slice locations based on the suspected area of acute edema or inflammation on cine images.

4. Use a thick (10-12 mm) slice.

5. Obtain short axis, mid ventricular slices by multiple breathholds, spoiled gradient echo sequences with nine echo time $(5.6-18 \mathrm{~ms})$ for $\mathrm{T} 2 *$ measurement for iron detection. Adjust the TR between the 9 RF (segment) pulses according to the different TE times.

\section{Valvular flow and shunt assessment module}

1. Quantitative flow measurement

i. For the flow velocity and volume, the plane of measurement should be perpendicular to the vessel and distal to valve leaflet tips of interest. Adapt velocity encoding to actual velocity by the lowest velocity without aliasing, and use the lowest TE possible for high velocity jet flows.

ii. Specific plane for phase contrast (PC) flow acquisition.

For the acquisition of different chamber views stated below, please refer to the basic modules on structure and function of LV and $\mathrm{RV}$ in Sections A \& B.

(a) Mitral Valve: Plan on the end-systolic two chamber and four chamber cine images. The center of the slice is placed in the middle of the mitral valve and angulated parallel to the mitral valve.

(b) Tricuspid Valve: Plan on true fourchamber and RV two-chamber images at the end systolic phase. The center of the slice is placed in the middle of the tricuspid valve and angulated parallel to the tricuspid valve.

(c) Aortic Valve: Alignment of the imaging plan for the aortic valve is from the 
three chamber and LVOT views. The imaging plane should be positioned just above the aortic valve, yet just below the origin of the coronary arteries. The LVOT view is prescribed perpendicular to the three chamber view.

(d) Pulmonary Valve: Align the imaging plane from two orthogonal RVOT views and place the plane just above the pulmonary valve. The first RVOT view is prescribed from an oblique plane through the main PA and RV on a set of axial images. The second RVOT plane is prescribed perpendicular to the first RVOT.

(e) Pulmonary Arteries: Main pulmonary artery (MPA)

Prescribe from sagittal localizer and obtain a few cine slices parallel to the MPA. The imaging plane for flow acquisition should be perpendicular to the direction of flow to the MPA on these images. The usual peak velocity is $180 \mathrm{~cm} / \mathrm{s}$. The encoding velocity recommended for the first measurement or for flow measurement is $180 \mathrm{~cm} / \mathrm{s}$.

Left pulmonary artery (LPA) and right pulmonary artery (RPA) Imaging planes through the LPA and RPA should be perpendicular to the direction of flow and proximal to the first branching vessel. In general, these planes are usually $1-1.5 \mathrm{~cm}$ distal to the MPA bifurcation. The usual velocity for peak measurement is $60-120 \mathrm{~cm} / \mathrm{s}$. The recommended encoding velocity for the first measurement or for flow measurement is $200 \mathrm{~cm} / \mathrm{s}$.

(f) Ascending Aorta: Prescribe the imaging plane from the coronal localizer. The flow acquisition is performed perpendicular to the flow direction of the ascending aorta, about $1.5-2 \mathrm{~cm}$ above the aortic valve at the pulmonary bifurcation level. The usual peak velocity measurement is $100-160 \mathrm{~cm} / \mathrm{s}$. The recommended encoding velocity for the first measurement or for flow measurement is $200 \mathrm{~cm} / \mathrm{s}$.
2. Qualitative assessment

i. Use gradient echo or hybrid gradient echo/ echo planar imaging as either is better in visualizing the severity of regurgitant jets or turbulence across shunts.

ii. For the morphology and planimetry of valve orifice, optimize the angle and level with SSFP in the plane of the valve of interest and use three contiguous $5 \mathrm{~mm}$-thick high resolution cines transecting the line of the jet and moving from orifice level to immediately downstream.

iii. Specific plane for assessment:
(a) Mitral valve: horizontal and vertical long axis, LVOT view
(b) Aortic valve: LVOT and coronal view
(c) Tricuspid valve: horizontal long axis and transaxial view
(d) Pulmonary valve: RV long axis or RVOT view

Recommended protocol for adenosine stress test

1. Follow the checklist attached and set up according to the monitoring sheet for adenosine stress myocardial perfusion test.

2. Perform LV structure and function module as listed or alternatively acquire these images at between stress and rest perfusion to shorten scanning time.

3. Start continuous adenosine infusion (at least $3 \mathrm{~min}$, preferably 4-6 min continuous infusion of $140 \mu \mathrm{g} / \mathrm{kg}$ body weight $/ \mathrm{min}$ ) and then

i. Perform the gadolinium first pass perfusion module; update the heart rate in response to the heart rate increase caused by adenosine.

ii. Gadolinium is injected $(0.05-0.1 \mathrm{mmol} / \mathrm{kg})$ during the last minute of adenosine infusion.

iii. Stop adenosine infusion after imaging for 50-60 heart beats when the gadolinium has passed through LV myocardium.

iv. Continuous ECG monitoring, oxygen saturation monitoring and repeated BP measured at baseline, during infusion, and for at least 2 min post-infusion of adenosine (see the monitoring sheet). 


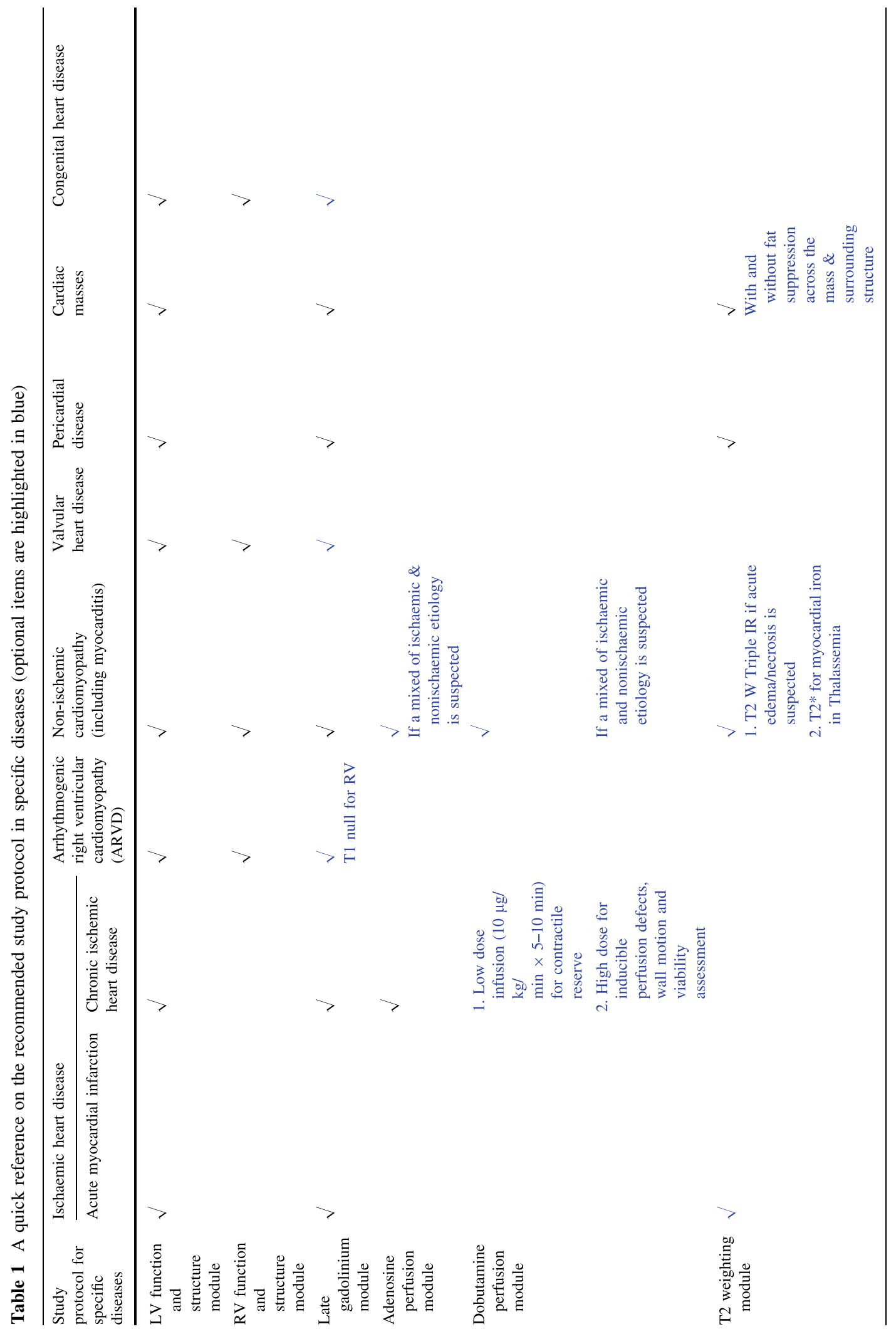




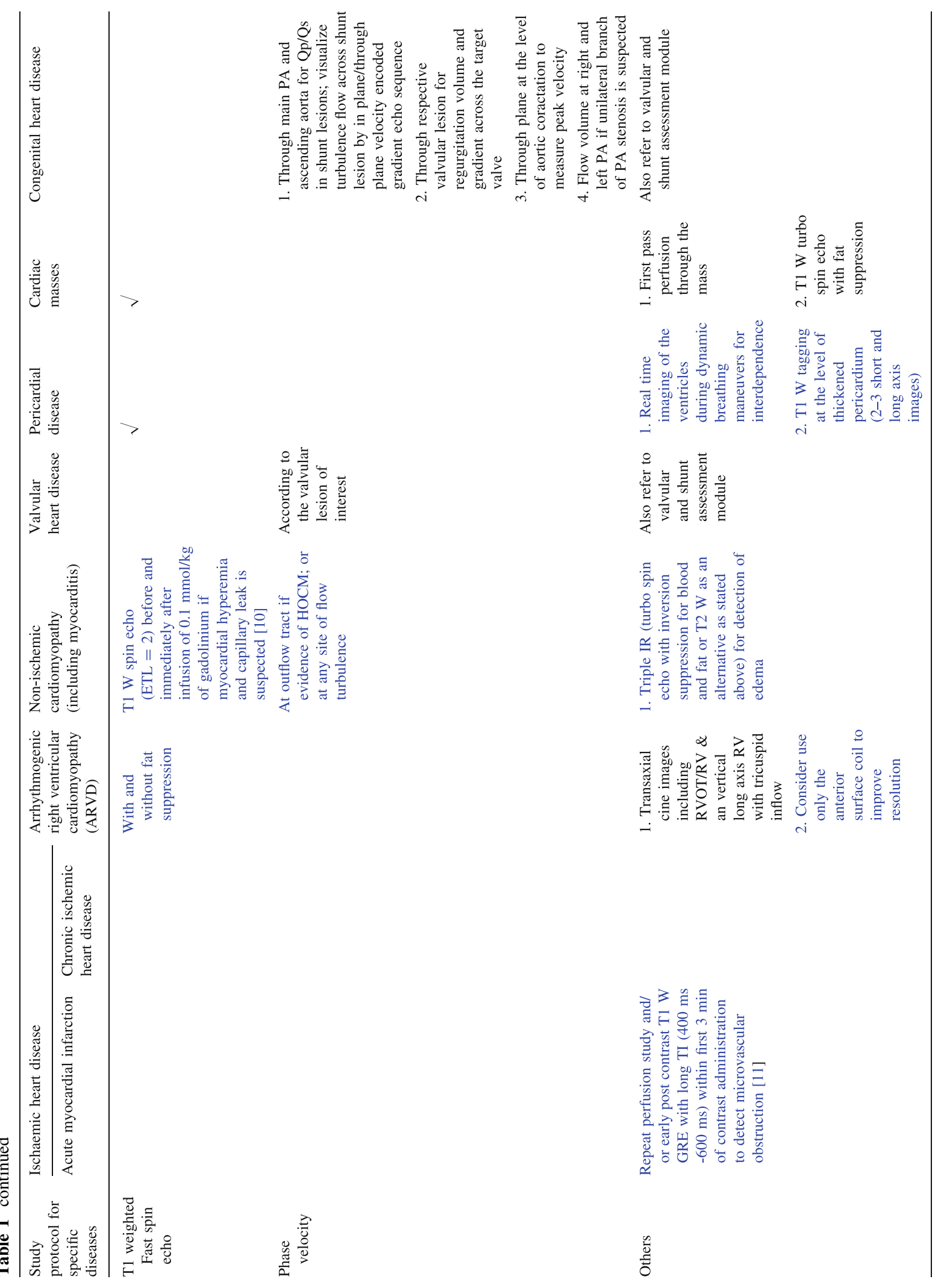




\section{Rest Perfusion}

i. Perform only after at least $10 \mathrm{~min}$ washout period for the gadolinium from stress perfusion imaging.

ii. Repeat perfusion imaging without adenosine using same dose of gadolinium and at the same slice location, again update the heart rate.

iii. Consider skipping if the stress perfusion is free from artifact. However, additional gadolinium may be considered for late gadolinium enhancement imaging (for a cumulative total dose of $0.1-0.2 \mathrm{mmol} / \mathrm{kg}$ ).

5. Perform the LGE module after waiting for about $10 \mathrm{~min}$ after the last dose of contrast.

Recommended protocol for dobutamine stress test

Low dose dobutamine infusion at $10 \mu \mathrm{g} / \mathrm{kg}$ for 5-10 $\mathrm{min}$ is an alternative to look for contractile reserve only.

1. Follow the attached checklist and set up according to the monitoring sheet for dobutamine stress myocardial perfusion test.

2. Follow baseline LV structure and function module.

3. Dobutamine continuous infusion:

i. Increase the dobutamine infusion in increments of $10 \mu \mathrm{g} / \mathrm{kg}$ body weight/minute every $3 \mathrm{~min}$ starting at $10 \mu \mathrm{g} / \mathrm{kg}$ body weight $/ \mathrm{min}$ until target heart rate $[85 \% \times(220$-age $)]$ is reached.

ii. Add atropine $0.3 \mathrm{mg}$ in fractional doses up to $2 \mathrm{mg}$, if heart rate response is poor.

iii. Repeat 3 short axis (basal, mid ventricular, and distal views) and 3 long axis cine views during each increment of dobutamine dosing.

iv. Continuously monitor ECG and oxygen, and measure $\mathrm{BP}$ at baseline, during each stage and at least $10 \mathrm{~min}$ after stress test (see the monitoring sheet).

v. View cine loops online as they are being acquired at each stage during real-time or almost at real-time.

vi. Optimize the temporal resolution at every dosage as the heart rate increases. vii. Stop test at detection of any new wall motion or thickening abnormality; systolic blood pressure increase $>240 \mathrm{mmHg}$ and/ or diastolic blood pressure $>120 \mathrm{mmHg}$; Blood pressure decrease $>20 \mathrm{mmHg}$ below baseline systolic blood pressure and/or decrease $>40 \mathrm{mmHg}$ from a previous level; severe chest pain or other intractable symptoms; complex cardiac arrhythmias or achievement of peak heart rate.

4. Perform first pass perfusion at peak stress with gadolinium at $0.05-0.075 \mathrm{mmol} / \mathrm{kg}$ as described in the perfusion module, wait for $20 \mathrm{~min}$ before acquisition of rest perfusion.

5. Perform the LGE module after waiting for at least $10 \mathrm{~min}$ after the last dose of gadolinium injection (at a cumulative dose of $0.1-0.2 \mathrm{mmol} /$ $\mathrm{kg})$.

\section{Conclusion}

The elements of the above practice guidelines recommended by ASCI are based on the currently available acquisition technology and knowledge on specific diseases. ASCI will continue to observe the field and provide the most up-to-date information to assist in the delivery of the best patient care (Table 1).

Acknowledgments The working group would like to acknowledge $\mathrm{Mr}$ Lawrance Yip, the senior radiographer, Queen Mary Hospital, Hong Kong for the acquisition of images and Dr Stephen CW Cheung, the consultant radiologist, Queen Mary Hospital, Hong Kong for the contribution on the worksheets for stress studies.

Declaration The members of the working group have nothing to declare on the potential conflict of interest.

Open Access This article is distributed under the terms of the Creative Commons Attribution Noncommercial License which permits any noncommercial use, distribution, and reproduction in any medium, provided the original author(s) and source are credited.

\section{Appendix}

See Tables 2, 3, 4, 5, 6, and 7 . 
Table 2 Checklist for adenosine stress myocardial test

Pre-procedure

I. Check for contraindication for adenosine stress test

1. Known hypersensitivity to adenosine

2. Known or suspected bronchoconstrictive or bronchospastic disease

3. Heart block more than first degree

4. Significant sinus bradycardia (resting heart rate $<45 \mathrm{bpm}$ )

5. Systemic arterial hypotension $(<90 \mathrm{mmHg})$ or severe arterial hypertension

6. Recent myocardial infarction within 3 days

II. Obtain informed consent

III. Perform pre-procedure 12 leads ECG and review by doctor in charge

IV. Place two angiocatheters intravenously to each arm:

1. One IV $20 \mathrm{G}$ in antecubital fossa for contrast

2. One IV 20-22G for adenosine infusion

V. Set up infusion pump for adenosine:

Drugs dosage: adenosine $0.14 \mathrm{mg} / \mathrm{kg} / \mathrm{min}$

Patient's body weight $(\mathrm{BW})=$

$\mathrm{kg}$; Adenosine concentration $\mathrm{mg} / \mathrm{ml}$

$0.140(\mathrm{mg} / \mathrm{kg} / \mathrm{min}) \times \mathrm{BW}(\mathrm{kg}) /$ Adenosine concentration $(\mathrm{mg} / \mathrm{ml})=$

Infusion rate $(\mathrm{ml} / \mathrm{min})=$ $(\mathrm{ml} / \mathrm{h})$

Total dose of adenosine to be administrated:

$0.14(\mathrm{mg} / \mathrm{kg} / \mathrm{min}) \times \mathrm{BW}(\mathrm{kg}) \times$ Infusion time $(\mathrm{min})=$ $\mathrm{mg}$

During-procedure

1. Oxygen $21 / \mathrm{min}$ per nasal cannula

2. Set BP cuff to manual mode. Place BP cuff opposite arm as med administration.

3. Record baseline vital signs- prior to medication administration

4. Aminophylline $125 \mathrm{mg}$ in $50 \mathrm{ml}$ normal saline IVB over 5-6 min prn chest pain and shortness of breath

5. Start adenosine infusion at $\mathrm{am} / \mathrm{pm}$ (time). Continue vital sign monitoring as shown

Post procedure

1. Perform post stress ECG and review

2. Record final vital signs prior to discharge. Report any patient complaints to doctor in charge 
Table 3 Patient monitoring during adenosine stress cardiac MR examination

\begin{tabular}{|c|c|c|c|c|c|}
\hline \multicolumn{2}{|c|}{$\begin{array}{l}\text { Time after start of } \\
\text { adenosine infusion }\end{array}$} & $\begin{array}{c}\text { Blood pressure } \\
(\mathrm{mmHg})\end{array}$ & Heart rate/min & $\mathrm{SaO2}$ reading $(\%)$ & Symptoms \\
\hline \multicolumn{6}{|c|}{ Baseline } \\
\hline \multicolumn{6}{|c|}{30 secs } \\
\hline \multicolumn{6}{|c|}{2 mins } \\
\hline \multicolumn{6}{|c|}{3.5 mins } \\
\hline \multicolumn{6}{|c|}{$\begin{array}{l}6 \text { mins } \\
\text { (intermediate after scan) }\end{array}$} \\
\hline \multicolumn{6}{|c|}{8 mins } \\
\hline \multicolumn{6}{|c|}{10 mins } \\
\hline \multicolumn{6}{|c|}{ Before discharge } \\
\hline \multicolumn{6}{|c|}{ Please indicate the reason(s) for premature termination: } \\
\hline$\square$ & Persistent or $\mathrm{s}$ & tic AV block & & & \\
\hline$\square$ & Significant dro & tolic blood pressure & & & \\
\hline$\square$ & Persistent or $\mathrm{s}$ & atic hypotension & & & \\
\hline$\square$ & Severe respira & culty & & & \\
\hline$\square$ & Others & & & & \\
\hline
\end{tabular}

Ref: potential side effects during adenosine infusion

1. Flushing, chest pain, palpitations and breathlessness

2. Transient heart block

3. Transient sinus tachycardia

4. Bronchospasm 
Table 4 Checklist for dobutamine stress myocardial test

Pre-procedure

Checked

I. Check for contraindication for dobutamine stress test

1. Severe systemic arterial hypertension $(\geq 220 / 120 \mathrm{mmHg})$

2. Unstable angina

3. High grade aortic stenosis (aortic valve area $<1 \mathrm{~cm} /$ peak aortic valve gradient $>50 \mathrm{mmHg}$ )

4. Uncontrolled or complex cardiac arrhythmias

5. Haemodynamically significant hypertrophic obstructive cardiomyopathy

6. Uncontrolled heart failure

7. Myocarditis, endocarditis and pericarditis

8. History of sudden death

9. Aortic dissection/high grade aortic aneurysm

10. Mobile left ventricular or left atrial thrombus

II. Check list for contraindications for atropine

1. Advance heart block

2. Glaucoma

3. Pyloric stenosis

4. Obstructive uropathy

5. Myasthenia gravis

II. Obtain informed consent

III. Perform pre-procedure 12 leads ECG and review by doctor in charge

IV. Place two angiocatheters intravenously to each arm:

1. One IV $20 \mathrm{G}$ in antecubital fossa for contrast

2. One IV 20-22G for adenosine infusion

V. Set up infusion pump for dobutamine infusion:

VI. Additional medication to be ready for use in examination room

1. TNG table

2. Metropolol $5 \mathrm{mg} / \mathrm{ml}$

3. Atropine $0.6 \mathrm{ml} \times 3$ ampules

4. NS flush

During-procedure

1. Oxygen 21 per nasal cannula

2. Set BP cuff to manual mode. Place BP cuff opposite arm as med administration.

3. Record baseline vital signs- prior to medication administration

4. Record time at the start of dobutmaine infusion. Continue vital sign monitoring whenever change of dosage and prn Post procedure

1. Perform post stress ECG and review

2. Record total amount of drugs given

3. Record final vital signs prior to discharge. Report any patient complaints to doctor in charge 
Table 5 Patient monitoring during dobutamine stress cardiac MR examination

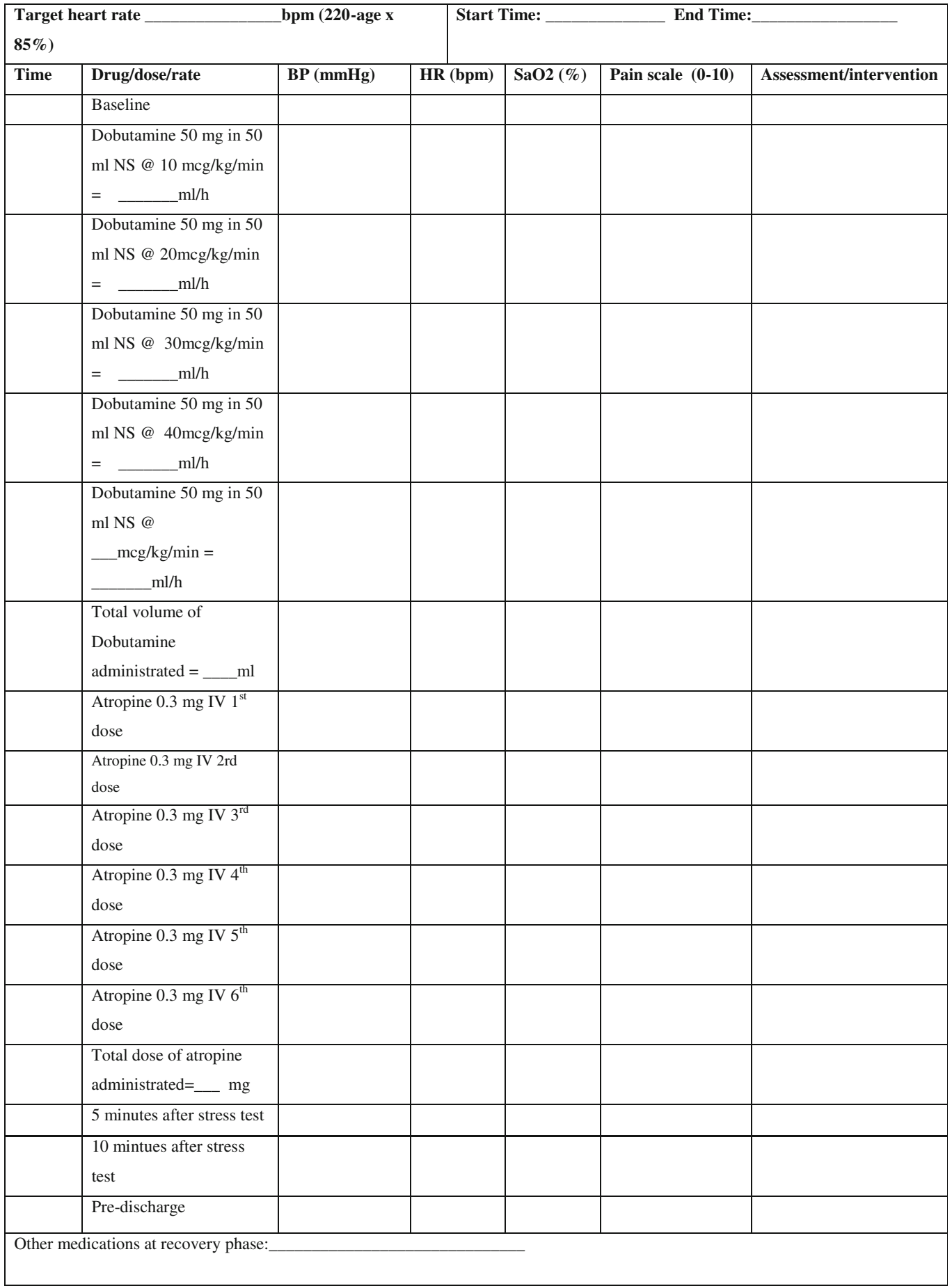

\section{Was target HR achieved: $\mathrm{Y} / \mathrm{N}$}

Reasons for termination:
$\square \quad$ Target heart rate
$\square \quad$ More than two sites wall motion abnormality
$\square \quad$ Severe chest pain
$\square \quad B P>220 / 120 \mathrm{mmHg}$
$\square \quad$ Arrhythmias
$\square$ Patient cannot tolerate due to other reasons

$\square \quad$ BP decrease $>20 \mathrm{mmHg}$ systolic below baseline systolic BP or decrease $>40 \mathrm{mmHg}$ form a previous level

$\square \quad$ Max dose of stress 
Table 6 Adenosine infusion protocol $(0.14 \mathrm{mg} / \mathrm{kg} / \mathrm{min}$ over $6 \mathrm{~min})$ adenosine concentration: $3 \mathrm{mg} / \mathrm{ml}$

\begin{tabular}{|c|c|c|c|c|c|c|c|c|c|}
\hline $\begin{array}{l}\mathrm{Wt} \\
(\mathrm{kg})\end{array}$ & $\begin{array}{l}\text { Wt } \\
(\mathrm{lbs})\end{array}$ & $\begin{array}{l}\text { Adenosine (mg/ } \\
\text { min) }\end{array}$ & $\begin{array}{l}\text { Adenosine } \\
(\mathrm{ml} / \mathrm{h})\end{array}$ & $\begin{array}{l}\text { Adenosine total } \\
\text { dose }\end{array}$ & $\begin{array}{l}\text { Wt } \\
(\mathrm{kg})\end{array}$ & $\begin{array}{l}\text { Wt } \\
(\mathrm{lbs})\end{array}$ & $\begin{array}{l}\text { Adenosine (mg/ } \\
\text { min) }\end{array}$ & $\begin{array}{l}\text { Adenosine } \\
(\mathrm{ml} / \mathrm{h})\end{array}$ & $\begin{array}{l}\text { Adenosine total } \\
\text { dose }\end{array}$ \\
\hline 40 & 88 & 5.6 & 112 & 34 & 75 & 165 & 10.5 & 210 & 63 \\
\hline 41 & 90 & 5.7 & 114 & 34 & 76 & 167 & 10.6 & 213 & 64 \\
\hline 42 & 92 & 5.9 & 118 & 35 & 77 & 169 & 10.8 & 216 & 65 \\
\hline 43 & 95 & 6.0 & 120 & 36 & 78 & 172 & 10.9 & 218 & 66 \\
\hline 44 & 97 & 6.1 & 122 & 37 & 79 & 174 & 11.1 & 221 & 66 \\
\hline 45 & 99 & 6.3 & 126 & 38 & 80 & 176 & 11.2 & 224 & 67 \\
\hline 46 & 101 & 6.4 & 129 & 39 & 81 & 178 & 11.3 & 227 & 68 \\
\hline 47 & 103 & 6.6 & 132 & 39 & 82 & 180 & 11.5 & 230 & 69 \\
\hline 48 & 106 & 6.7 & 134 & 40 & 83 & 183 & 11.6 & 232 & 70 \\
\hline 49 & 108 & 6.9 & 137 & 41 & 84 & 185 & 11.8 & 235 & 71 \\
\hline 50 & 110 & 7.0 & 140 & 42 & 85 & 187 & 11.9 & 238 & 71 \\
\hline 51 & 112 & 7.1 & 143 & 43 & 86 & 189 & 12.0 & 241 & 72 \\
\hline 52 & 114 & 7.3 & 146 & 44 & 87 & 191 & 12.2 & 244 & 73 \\
\hline 53 & 117 & 7.4 & 148 & 45 & 88 & 194 & 12.3 & 246 & 74 \\
\hline 54 & 119 & 7.6 & 151 & 45 & 89 & 196 & 12.5 & 249 & 75 \\
\hline 55 & 121 & 7.7 & 154 & 46 & 90 & 198 & 12.6 & 252 & 76 \\
\hline 56 & 123 & 7.8 & 157 & 47 & 91 & 200 & 12.7 & 255 & 76 \\
\hline 57 & 125 & 8.0 & 160 & 48 & 92 & 202 & 12.9 & 258 & 77 \\
\hline 58 & 128 & 8.1 & 162 & 49 & 93 & 205 & 13.0 & 260 & 78 \\
\hline 59 & 130 & 8.3 & 165 & 50 & 94 & 207 & 13.2 & 263 & 79 \\
\hline 60 & 132 & 8.4 & 168 & 50 & 95 & 209 & 13.3 & 266 & 80 \\
\hline 61 & 134 & 8.5 & 171 & 51 & 96 & 211 & 13.4 & 269 & 81 \\
\hline 62 & 136 & 8.7 & 174 & 52 & 97 & 213 & 13.6 & 272 & 81 \\
\hline 63 & 139 & 8.8 & 176 & 53 & 98 & 216 & 13.7 & 274 & 82 \\
\hline 64 & 141 & 9.0 & 179 & 54 & 99 & 218 & 13.9 & 277 & 83 \\
\hline 65 & 143 & 9.1 & 182 & 55 & 100 & 220 & 14.0 & 280 & 84 \\
\hline 66 & 145 & 9.2 & 185 & 55 & 101 & 222 & 14.1 & 283 & 85 \\
\hline 67 & 147 & 9.4 & 188 & 56 & 102 & 224 & 14.3 & 286 & 86 \\
\hline 68 & 150 & 9.5 & 190 & 57 & 103 & 227 & 14.4 & 288 & 87 \\
\hline 69 & 152 & 9.7 & 193 & 58 & 104 & 229 & 14.6 & 291 & 87 \\
\hline 70 & 154 & 9.8 & 196 & 59 & 105 & 231 & 14.7 & 294 & 88 \\
\hline 71 & 156 & 19.9 & 199 & 60 & 106 & 233 & 14.8 & 297 & 89 \\
\hline 72 & 158 & 10.1 & 202 & 60 & 107 & 235 & 15.0 & 300 & 90 \\
\hline 73 & 161 & 10.2 & 204 & 61 & 108 & 238 & 15.1 & 302 & 91 \\
\hline 74 & 163 & 10.4 & 207 & 62 & 109 & 240 & 15.3 & 305 & 92 \\
\hline
\end{tabular}


Table 7 Adenosine infusion protocol $(0.14 \mathrm{mg} / \mathrm{kg} / \mathrm{min}$ over $6 \mathrm{~min})$ adenosine concentration: $2 \mathrm{mg} / \mathrm{ml}$

\begin{tabular}{|c|c|c|c|c|c|c|c|c|c|}
\hline $\begin{array}{l}\text { Wt } \\
(\mathrm{kg})\end{array}$ & $\begin{array}{l}\text { Wt } \\
(\mathrm{lbs})\end{array}$ & $\begin{array}{l}\text { Adenosine (mg/ } \\
\text { min) }\end{array}$ & $\begin{array}{l}\text { Adenosine } \\
(\mathrm{ml} / \mathrm{h})\end{array}$ & $\begin{array}{l}\text { Adenosine total } \\
\text { dose }\end{array}$ & $\begin{array}{l}\text { Wt } \\
(\mathrm{kg})\end{array}$ & $\begin{array}{l}\text { Wt } \\
(\mathrm{lbs})\end{array}$ & $\begin{array}{l}\text { Adenosine }(\mathrm{mg} / \\
\mathrm{min})\end{array}$ & $\begin{array}{l}\text { Adenosine } \\
(\mathrm{ml} / \mathrm{h})\end{array}$ & $\begin{array}{l}\text { Adenosine total } \\
\text { dose }\end{array}$ \\
\hline 40 & 88 & 5.6 & 168 & 34 & 75 & 165 & 10.5 & 315 & 63 \\
\hline 41 & 90 & 5.7 & 171 & 34 & 76 & 167 & 10.6 & 318 & 64 \\
\hline 42 & 92 & 5.9 & 177 & 35 & 77 & 169 & 10.8 & 324 & 65 \\
\hline 43 & 95 & 6.0 & 180 & 36 & 78 & 172 & 10.9 & 327 & 66 \\
\hline 44 & 97 & 6.1 & 183 & 37 & 79 & 174 & 11.1 & 333 & 66 \\
\hline 45 & 99 & 6.3 & 189 & 38 & 80 & 176 & 11.2 & 336 & 67 \\
\hline 46 & 101 & 6.4 & 192 & 39 & 81 & 178 & 11.3 & 339 & 68 \\
\hline 47 & 103 & 6.6 & 198 & 39 & 82 & 180 & 11.5 & 345 & 69 \\
\hline 48 & 106 & 6.7 & 201 & 40 & 83 & 183 & 11.6 & 348 & 70 \\
\hline 49 & 108 & 6.9 & 207 & 41 & 84 & 185 & 11.8 & 354 & 71 \\
\hline 50 & 110 & 7.0 & 210 & 42 & 85 & 187 & 11.9 & 357 & 71 \\
\hline 51 & 112 & 7.1 & 213 & 43 & 86 & 189 & 12.0 & 360 & 72 \\
\hline 52 & 114 & 7.3 & 219 & 44 & 87 & 191 & 12.2 & 366 & 73 \\
\hline 53 & 117 & 7.4 & 222 & 45 & 88 & 194 & 12.3 & 369 & 74 \\
\hline 54 & 119 & 7.6 & 228 & 45 & 89 & 196 & 12.5 & 375 & 75 \\
\hline 55 & 121 & 7.7 & 231 & 46 & 90 & 198 & 12.6 & 378 & 76 \\
\hline 56 & 123 & 7.8 & 234 & 47 & 91 & 200 & 12.7 & 381 & 76 \\
\hline 57 & 125 & 8.0 & 240 & 48 & 92 & 202 & 12.9 & 387 & 77 \\
\hline 58 & 128 & 8.1 & 243 & 49 & 93 & 205 & 13.0 & 390 & 78 \\
\hline 59 & 130 & 8.3 & 249 & 50 & 94 & 207 & 13.2 & 396 & 79 \\
\hline 60 & 132 & 8.4 & 252 & 50 & 95 & 209 & 13.3 & 399 & 80 \\
\hline 61 & 134 & 8.5 & 255 & 51 & 96 & 211 & 13.4 & 402 & 81 \\
\hline 62 & 136 & 8.7 & 261 & 52 & 97 & 213 & 13.6 & 408 & 81 \\
\hline 63 & 139 & 8.8 & 264 & 53 & 98 & 216 & 13.7 & 411 & 82 \\
\hline 64 & 141 & 9.0 & 270 & 54 & 99 & 218 & 13.9 & 417 & 83 \\
\hline 65 & 143 & 9.1 & 273 & 55 & 100 & 220 & 14.0 & 420 & 84 \\
\hline 66 & 145 & 9.2 & 276 & 55 & 101 & 222 & 14.1 & 423 & 85 \\
\hline 67 & 147 & 9.4 & 282 & 56 & 102 & 224 & 14.3 & 429 & 86 \\
\hline 68 & 150 & 9.5 & 285 & 57 & 103 & 227 & 14.4 & 432 & 87 \\
\hline 69 & 152 & 9.7 & 291 & 58 & 104 & 229 & 14.6 & 438 & 87 \\
\hline 70 & 154 & 9.8 & 294 & 59 & 105 & 231 & 14.7 & 441 & 88 \\
\hline 71 & 156 & 19.9 & 597 & 60 & 106 & 233 & 14.8 & 444 & 89 \\
\hline 72 & 158 & 10.1 & 303 & 60 & 107 & 235 & 15.0 & 450 & 90 \\
\hline 73 & 161 & 10.2 & 306 & 61 & 108 & 238 & 15.1 & 453 & 91 \\
\hline 74 & 163 & 10.4 & 312 & 62 & 109 & 240 & 15.3 & 459 & 92 \\
\hline
\end{tabular}




\section{References}

1. American Heart Association (2009) International cardiovascular disease statistics. Available at http://www.americanheart. org/downloadable/heart/1236204012112INTL.pdf. Accessed on 6 June 2010

2. Modell B, Darlison M (2008) Global epidemiology of haemoglobin disorders and derived service indicators. Bull World Health Organ 86(6):480-487

3. Hendel RC, Budoff MJ, Cardella JF et al (2009) ACC/ AHA/ACR/ASE/ASNC/HRS/NASCI/RSNA/SAIP/SCAI/ SCCT/SCMR/SIR 2008 Key data elements and definitions for cardiac imaging: A report of the American college of cardiology/American heart association task force on clinical data standards (writing committee to develop clinical data standards for cardiac imaging). Circulation 119(1): 154-186

4. Hendel RC, Patel MR, Kramer CM et al (2006) ACCF/ ACR/SCCT/SCMR/ASNC/NASCI/SCAI/SIR 2006 appropriateness criteria for cardiac computed tomography and cardiac magnetic resonance imaging: a report of the American college of cardiology foundation quality strategic directions committee appropriateness criteria working group, American college of radiology, society of cardiovascular computed tomography, Society for cardiovascular magnetic resonance, American society of nuclear cardiology, North American society for cardiac imaging, society for cardiovascular angiography and interventions, and society of interventional radiology. J Am Coll Cardiol 48(7):1475-1497
5. Kramer CM, Barkhausen J, Flamm SD et al (2008) Standardized cardiovascular magnetic resonance imaging (CMR) protocols, society for cardiovascular magnetic resonance: board of trustees task force on standardized protocols. J Cardiovasc Magn Reson 10:35

6. Cerqueira MD, Weissman NJ, Dilsizian V et al (2002) Standardized myocardial segmentation and nomenclature for tomographic imaging of the heart: a statement for healthcare professional from the cardiac imaging committee of the council on clinical cardiology of the American Heart Association. Circulation 105(4):539-542

7. Todd D, Lai MC, Braga CA et al (1969) Alpha-thalassaemia in Chinese: cord blood studies. Br J Haematol 16(6):551-556

8. Li AM, Lee FT, Todd D (1982) The screening of Chinese cord blood for haemoglobinopathies. Hum Hered 32(1): $62-70$

9. Ghosh A, Woo JS, Wan CW et al (1985) Evaluation of a prenatal screening procedure for beta-thalassaemia carriers in a Chinese population based on the mean corpuscular volume (MCV). Prenat Diagn 5(1):59-65

10. Friedrich MG, Sechtem U, Schulz-Menger J et al (2009) Cardiovascular magnetic resonance in myocarditis: a JACC white paper. J Am Coll Cardiol 53(17):1475-1487

11. Mather AN, Lockie T, Nagel E et al (2009) Appearance of microvascular obstruction on high resolution first-pass perfusion, early and late gadolinium enhancement CMR in patients with acute myocardial infarction. J Cardiovasc Magn Reson 11:33 